\section{$\underset{\text { hommes }}{\text { \& migrations }}$}

\section{Hommes \& migrations}

Revue française de référence sur les dynamiques

migratoires

$1310 \mid 2015$

Fashion Mix

\title{
Les aventuriers de la mode
}

Les sapeurs congolais à Paris et l'usage de la mode en migration

(1890-2014)

\section{Manuel Charpy}

\section{(2) OpenEdition \\ Journals}

Édition électronique

URL : http://journals.openedition.org/hommesmigrations/3146

DOI : 10.4000/hommesmigrations.3146

ISSN : 2262-3353

Éditeur

Musée national de l'histoire de l'immigration

Édition imprimée

Date de publication : 1 avril 2015

Pagination : 25-33

ISBN : 978-2-919040-31-5

ISSN : $1142-852 X$

Référence électronique

Manuel Charpy, «Les aventuriers de la mode », Hommes \& migrations [En ligne], 1310 | 2015, mis en ligne le 01 avril 2018, consulté le 01 mai 2019. URL : http://journals.openedition.org/ hommesmigrations/3146; DOI : 10.4000/hommesmigrations.3146 


\title{
LES AVENTURIERS DE LA MODE \\ LES SAPEURS CONGOLAIS À PARIS ET L'USAGE DE LA MODE EN MIGRATION (1890-2014)
}

Par MANUEL CHARPY, historien, chargé de recherche au CNRS / IRHIS, université de Lille-III.

\author{
Les sapeurs congolais sont passés maîtres dans l'art \\ du retournement des symboles. Ceux de la mode et ceux \\ des sociétés dans lesquelles ils s'affichent. Entre Paris \\ et Brazzaville, leur volonté de paraître n'est pas du goût de \\ tous. Ainsi, vêtements, poses théâtrales et "danse des griffes" \\ prennent à revers les attentes qui pèsent sur l'immigré \\ ou le Congolais de retour au pays. Le sapeur se plaît à faire \\ de la mode un jeu de miroirs décevant tout stéréotype.
}

Lors du défilé féminin printemps-été 2010 de Paul Smith surgit une mannequin noire, chapeau melon et gilet orange, costume rose, pantalon qui dévoile chaussettes et chaussures rouges. L'assistance sourit de ce mannequin qui rappelle confusément les nègres trop habillés de Tintin au Congo et, avec ses lèvres surlignées en fuchsia, les minstrels, ces blancs grimés qui autour de 1900 caricaturaient les Noirs ${ }^{1}$. Opportunément, l'arrivée d'une mannequin blanche, vêtue et grimée de la même manière, évite que le malaise ne s'installe.

Ces détournements sont ordinaires dans une industrie de la mode qui se régénère dans les marges - et quoi de plus marginal que les sapeurs, ces élégants originaires du Congo qui défilent à Brazzaville ou dans les rues du XVIII arrondissement de Paris ? Quelques photographies de Tamagni et Médiavilla ont suffi pour que Paul Smith se saisisse de ces excentricités, vidées de leur sens, la mode digérant sa propre critique. L'opération est d'autant plus vertigineuse que les sapeurs eux-mêmes ne cessent de braconner, en maîtres du détournement, sur les terres du luxe pour reconstruire une culture fondée sur l'apparence et les pratiques vestimentaires ${ }^{2}$. Le Bachelor, dans sa boutique Connivences de la Goutte-d'Or, ne s'en inquiète pas, habitué 
qu'il est aux récupérations - soirées branchées $^{3}$, danse contemporaine ${ }^{4}$, bière irlandaise $^{5}$... - qu'il voit comme des hommages et une publicité. Arrivé à Paris en 1977, il incarne la génération des "aventuriers", ou mikilistes - ceux qui ont vu le monde -, ces jeunes congolais partis pour Paris. Il est une des figures de la Sape, la Société des ambianceurs et des personnes élégantes, aussi select qu'informelle. Elle présente toutes les caractéristiques d'une subculture : groupe réduit - moins d'un millier de sapeurs à Paris et quelques centaines dispersés entre Bruxelles, Nantes et Lyon ${ }^{6}$-, construit autour de consommations culturelles élaborées en marge de la culture dominante - même s'ils jouent avec elle -, territoires singuliers, codes vestimentaires et gestuelles propres, série de règles implicites et explicites, langue ${ }^{7} .$.

Mais les sapeurs déjouent cette lecture des sphères culturelles. La Sape existe et se caractérise par une apparente surPortrait de Matsoua promené par des disciples, Congo, 2011. (c) D.R. adhésion à la culture dominante, par le fétichisme des grandes marques et par le jeu avec les signes de la réussite sociale ${ }^{8}$. Après un temps fort au début des années 1980 - ce dont témoigne la remarquable étude de JustinDaniel Gandoulou ${ }^{9}-$, elle a été donnée pour obsolescente, mouvement s'évanouissant avec la jeunesse de ses acteurs. Mais la résurgence médiatique des années 2000 a rappelé que le mouvement était encore vivace ${ }^{10}$. Si la Sape ne cesse de renaître, c'est qu'elle est liée à une situation politique, sociale et culturelle et qu'elle prend racine dans une histoire longue ; loin d'un mouvement sans mémoire, elle est le produit de la situation coloniale puis de l'immigration.

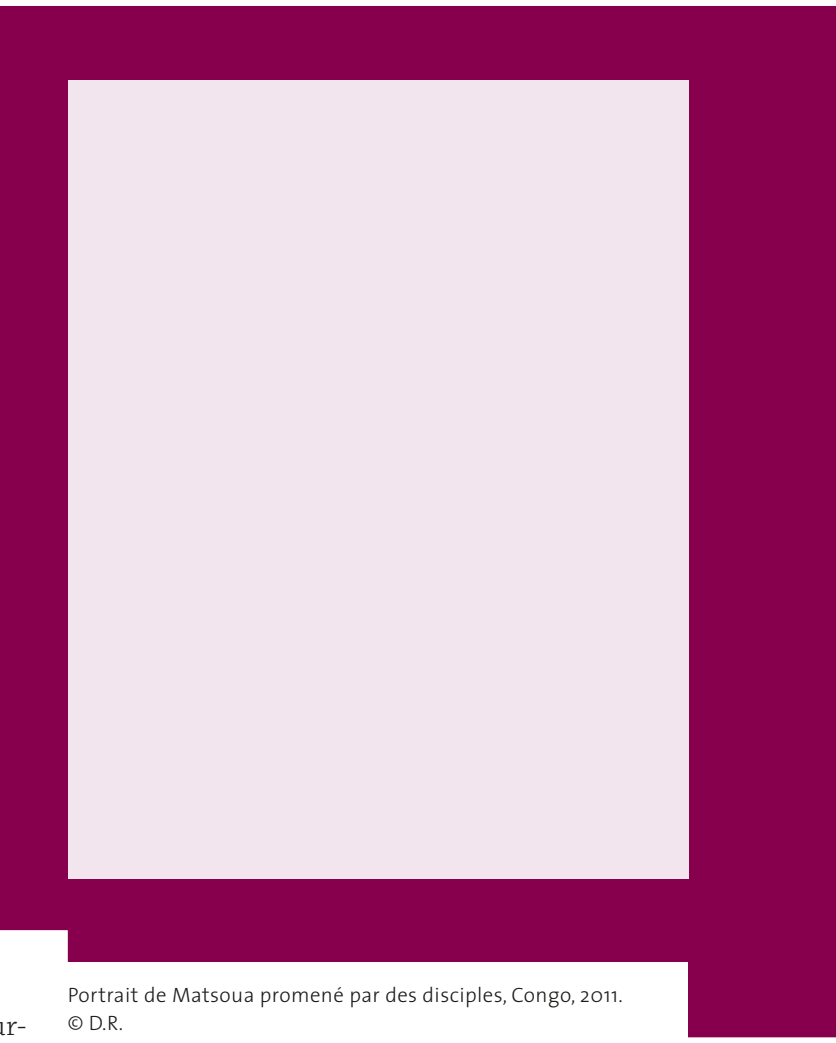

\section{Saper la colonie?}

À écouter les sapeurs sur leurs origines, le dandysme vient au premier plan. Cette filiation avec des manières scandaleuses d'échapper aux réalités prosaïques est volontiers reprise par la presse et les études sociologiques. Vêtements élégants et volontiers britanniques - kilts, cravates à la Byron, deerstalkers... -, gestuelle sophistiquée, distance avec le travail physique et dépenses ostentatoires : tout accrédite cette généalogie. Pourtant, une dif-

3. "Du Billisme à la sape", in Comptoir Général, 11 février 2012. 4. Coup fatal, direction artistique : Alain Platel, 2014.

5. Guiness, agence AMV BBDO, 2014. 6. Les Congolais étaient seulement 1172 en 1968, 8940 en 1982 et 12755 en 1990 dont $90 \%$ d'hommes célibataires. Voir Jean-Baptiste Douma, L'Immigration congolaise en France. Entre crises et recherche d'identité, Paris, L'Harmattan, 2003. 7. Dick Hebdige, Le Sens du style, Paris, Zones, 2008 [1979]. 8. Pierre Bourdieu, Yvette Delsaut, "Le couturier et sa griffe : contribution à une théorie de la magie", in Actes de la recherche en sciences sociales, $n^{\circ} 1$, janvier 1975 , pp. 7-36. 9. Justin Daniel Gandoulou, Entre Paris et Bacongo, Paris, Centre Georges-Pompidou / CCl, 1984. Voir, en complément, Charles-Didier Gondola, “La sape des mikilistes : théâtre de l'artifice et représentation onirique”, in Cahiers d'études africaines, $n^{\circ} 153,1999$, pp. 13-47. Notre travail repose en outre sur des enquêtes et entretiens menés entre novembre 2009 et novembre 2014. 10. Alain Mabanckou, Black Bazar, Paris, Seuil, 2009, et Frédéric Ciriez, Mélo, Paris, Verticales, 2013. 
férence radicale demeure : s'il refuse travail et épargne, le dandy dilapide le plus souvent la fortune familiale ${ }^{11}$. Les sapeurs sont loin de cette réalité, et c'est justement cet écart qui leur fait revendiquer cette filiation. Ce récit est d'abord construit pour se faire entendre de la société française. En réalité, leurs pratiques subversives du vêtement naissent avant les migrations, en situation coloniale.

Dans la pelote de leurs récits, une des généalogies revendiquées est celle avec Matsoua. Vivant à Bacongo dans les années 1920, il rejoint les tirailleurs sénégalais en 1925 puis s'installe à Paris où il fréquente les cercles anticoloniaux et fonde l'Amicale, base du mouvement anticolonial congolais. Déporté au Tchad, il s'échappe et rejoint Paris avant de s'engager dans l'armée en 1939. Arrêté et extradé au Congo en 1940, il meurt dans des conditions obscures en 1942. Naît alors le matsouanisme, courant politico-religieux qui voit en lui un messie $^{12}$. Les sapeurs s'en réclament, soulignant qu'il revint au pays dans un uniforme impeccable. Double légitimité, politique et historique, par l'inscription dans une tradition, les sapeurs affirmant volontiers que leurs "parents" se sapaient. S'ils n'évoquent presque jamais le billisme ${ }^{13}$, ils se rattachent volontiers à l'indépendance où la libération touche aussi les corps. Mais le pays entre vite en crise. Le régime de Fulbert Youlou - "l'homme aux soutanes Dior" - et le "socialisme scientifique" des années 1960-1990 sont peu propices à la Sape. Matsoua n'en est que plus revendiqué, notamment par les sapeurs qui partent en France. En désignant ce fondateur, ils réactivent une longue mémoire faite de gestes, de pratiques vestimentaires et de récits anticoloniaux, mettant en miroir le statut du colonisé et de l'immigré ${ }^{14}$.

\section{Le retournement des symboles de l'ordre colonial}

Dès les débuts de la colonisation dans les années 1880, les témoins raillent les "nègres" qui s'emparent de vêtements européens ${ }^{15}$. Les récits de Stanley sont fondateurs : il décrit les chefs portant "une livrée bleue de domestique, un bonnet phrygien en tricot multicolore et un caleçon de nuance criarde" ou "vêtus d'une tunique rouge de soldat anglais, d'un chapeau de feutre brun, d'un caleçon à carreaux ${ }^{16 "}$. Quand on rapporte le voyage de Savorgnan de Brazza, le roi de Sogno est campé avec "un chapeau de gendarme (...), un pagne aux vives couleurs, une redingote noire ${ }^{17}$. Le motif devient pon-

cif. En 1898, Pierre Verhaegen relève ainsi "les accoutrements les plus étranges" portés "d'un air superbe" et raille à Tumba un roi avec "un ancien uniforme de l'armée anglaise (...);
Dès les débuts de la colonisation dans les années 1880 , les témoins raillent les "nègres" qui s'emparent de vêtements européens. de carabinier d'Offenbach ${ }^{18 "}$. Ces pratiques n'inquiètent pas, les rois affublés d'oripeaux apparaissant d'opérette. Mais elles se diffusent du fait de l'importation de fripes européennes.

Le Congo français note: "Ce que la côte occidentale d'Afrique consomme de vieux habits, de vestons passés, de redingotes usées, de fracs hors d'usage, de tuniques d'uniformes démodées, est inimaginable. Les anciens uniformes rouges ou bleus des soldats anglais ou français trouvent là un placement admirable. (...) Il n'est pas de frac, quelque usé qu'il puisse être, qui ne trouve amateur au Congo ${ }^{19}$." Et un lieutenant d'espérer parvenir "à décider les millions de nègres de l'intérieur à se

11. Colin Campbell, The Romantic Ethic and the Spirit of Modern Consumeris, Oxford, Basil Blackwell, 1987.

12. Voir Martial Sinda, Le Messianisme congolais et ses incidences politiques..., Paris, Payot, 1972 et André Matsoua : fondateur du mouvement de libération du Congo, Paris/Abidjan/Dakar, Afrique Biblio Club, 1977. 13. Guillaume Godard, "Le billisme", photographies de Jean Depara, in Feuilleton, $\mathrm{n}^{\circ}$ 2, hiver 2012. 14. Sur cette réactivation par les populations déportées aux États-Unis comme esclaves et les générations suivantes, voir Monica Miller, Slaves to Fashion. Black Dandyism and the Styling of Black Diasporic Identity, Durham, Duke University Press, 2009. 15. Sur cette approche, essentiellement sur le XX siècle, voir Phyllis M. Martin, “Contesting clothes in colonial Brazzaville”, in The Journal of African History, vol. 35, $n^{\circ} 3,1994, p p .401-426$, et Jean Comaroff, "The Empire's old clothes: fashioning the colonial subject", in David Howes (dir.), Cross Cultural Consumption: Global Markets and Local Realities, Londres, Routledge, 1996. 16. Henry M. Stanley, Cing années au Congo :1879-1884, Paris, Dreyfous,1885, pp. 84-85. 17. Didier Neuville, Charles Bréard, Les Voyages de Savorgnan de Brazza. Ogôoué et Congo (1875-1882), Paris, Berger-Levrault,1884, p. 181. 18. Pierre Verhaegen, Au Congo. Impressions de voyage, Gand, Siffer, 1898, pp. 30-100. 
dépouiller de leur accoutrement d'herbes sèches, pour adopter des vêtements d'occasion européens", y compris les "chefs du Congo, qui s'y pavaneraient avec joie, les jours où ils auraient à se mettre en grande tenue $e^{20 "}$. Et de conclure à propos d'un "chef casqué" qu'il est "l'esclave de la mode". Si le roi en vieille redingote bourgeoise fait rire, les domestiques et commerçants qui s'habillent élégamment inquiètent de plus en plus tant la pratique, incontrôlable, remet en cause les hiérarchies du système colonial. On peut écrire alors que "les Noirs de Sierra Leone sont encore plus insupportables que les électeurs de Dakar.

Ils sont tous habillés aux dernières

Les aventuriers portent

ce renversement

dans les années 1980,

Djo Balard arpente Paris

en bermuda - japonais -

et coiffé d'un casque

colonial. modes de chez nous et se promènent, pour célébrer le dimanche anglican, en redingote par 38 degrés à l'ombre, en haut-de-forme ${ }^{21}$ ". Le phénomène est encore plus marqué au Congo où on note en 1913 : “Aujourd'hui les indigènes de la région de Brazzaville ne s'habillent que trop et, le dimanche, ceux qui possèdent plusieurs pantalons, plusieurs paletots, mettent ces vêtements les uns par dessus les autres (...). Beaucoup se piquent de suivre la mode parisienne, et, ayant su que naguère les Européens plaisantaient sur la passion des Noirs pour les chapeaux haut de forme, [ils] arborent aujourd'hui d'élégants panamas 22." Durant les années 1920, l'administration ne cesse de surveiller ces pratiques, d'autant que des mouvements anticoloniaux ailleurs usent aussi du vêtement ${ }^{23}$. Les années 1930-1950 sont tissées de ces descriptions, de Tintin au Congo ${ }^{24}$ (1931), où sont ridiculisés des Noirs portant feutres, cravates, cols et manchettes, à Gide qui décrit un chef "en redingote très longue et très fripée, casquette kaki, culotte kaki, leggins noirs, gros souliers ferrés. Le tout ineffablement laid et ridicule 25 ". Quand les "aventuriers” partent pour Paris, dans les années 1960, c'est avec ces discours en tête. Double héritage donc : ils prolongent des pratiques subversives et portent le poids de ces représentations qui font que soit ils s'habillent trop peu et sont assimilés à des "sauvages", soit ils s'habillent et sont regardés comme naïvement déguisés.

Les aventuriers portent ce renversement : dans les années 1980, Djo Balard arpente Paris en bermuda - japonais - et coiffé d'un casque colonial ${ }^{26}$. Et les sapeurs jouent volontiers avec des uniformes dont on ne sait pas s'ils sont d'Ancien Régime ou coloniaux. Rien de surprenant à voir Connivences proposer des sahariennes, vêtement à la fois des militaires anglais et dédié aux safaris, pour des immigrés en "aventure". Les sapeurs renvoient ainsi la société française à son passé honteux. Longtemps, leur lieu de rendezvous était "les chaînes", celles entourant la place de la République. Le terme évoque ironiquement la chaîne de montage mais aussi l'esclavage colonial, les renvoyant dos à dos. Et quand quelques sapeurs pratiquent l'escroquerie au chèque, ils l'appellent la "dette coloniale". Par les mots et les vêtements, ils revendiquent ainsi un héritage anticolonial, manière de saper la domination sociale et culturelle en migration.

\section{"Mon corps ne supporte pas le jean" ou l'impossible main-d'œuvre}

Une autre histoire rattachela Sape aux migrations. La notice Wikipédia, rédigée en partie par des sapeurs, en donne un résumé : "L'inventeur du mot "Sape" serait Christian Loubaki dit enfant Mystère. Cet homme à tout faire travaillait chez des aristocrates français dans le quartier huppé du XVIe arrondissement (...). En 1975, son patron lui aurait dit :

19. Alexis Marie Gochet, Le Congo français illustré, Paris, Procure générale, 189o, p. 155 et sq. 20. Lieutenant Lemaire, Au Congo. Comment les Noirs travaillent, Bruxelles, Bulens, 1895, pp. 104-107. 21. L'Expansion belge, vol. 3, 1910, p. 348. 22. Baron de Witte, Les Deux Congos, Paris, Plon, 1913. 23. ANOM 5D88 5 D 88, Mouvement Balali (1929-1930); affaire Grenard; affaire Bima ; incidents de Brazzaville. Voir par exemple, C. A. Bayly, "The origins of Swadeshi: cloth and Indian society, 1700-1930", in Arjun Appadurai (dir.), The Social Life of Things, Cambridge, Cambridge University Press, 1986, pp. 285-322, et Nira Wickramasinghe, Dressing the Colonised Body: Politics, Clothing and Identity in Colonial Sri Lanka, New Delhi, Longman, 2003. 24. Hergé, "Tintin au Congo " in Petit Vingtième, supplément du quotidien belge conservateur Le Vingtième siècle, juin 1930-juin 1931. 25. André Gide, Voyage au Congo, Paris, Gallimard, 1927. 
Doublure en wax et doublure bleu-blanc-rouge, vestes Connivences, 2009. @ Manuel Charpy, KeVIn NGomsik et Pablo Grand-Mourcel

'Tel que tu t'es habillé, tu vas saper le moral de tes amis.' [Il] a ainsi affirmé qu'il était le mieux sapé !" Cette histoire souligne le renversement social opéré par les sapeurs et les inscrit dans l'histoire de l'immigration. De fait, la Sape désignée comme telle advient avec l'immigration et sa reconfiguration suite à la fin des norias. Dès lors, les jeunes Congolais, même étudiants, sont assimilés à de la "main-d'œuvre immigrée", marginale et précaire, "Parisiens refoulés" dit une chanson des sapeurs. Les sapeurs refusent ce statut. Leurs vêtements, souliers vernis et costumes ajustés au corps, disent le refus, comme une application des théories de Veblen, du travail manuel ${ }^{27}$. Les vêtements accompagnent une démarche sophistiquée et lente qui se distingue du déplacement utile et efficace du travailleur : les sapeurs enchaînent "pas perdus", "diatances", "demi-tours complets", "chancèlements ${ }^{28}$ "... En ce sens, ils sont proches des zooteurs, Afro-Américains et immigrés qui répondent à leur marginalisation par des vêtements qui empêchent le travail ${ }^{29}$. Dans les années 1980, les sapeurs disent crânement ce mépris : "Il y a à Paris des Africains qui font honte (les Maliens)

26. Thomas Gilou, Black Mic-Mac, 1986, et entretiens, 2009-2011. 27. Thorstein Veblen, Théorie de la classe de loisir, Paris, Gallimard, 1970 [1899]. 28. "Sapologie, anthologie d'une culture urbaine made in Congo", in vybesnation.com, le 23 avril 2014 29. Kathy Peiss, Zoot Suit. The Enigmatic Career of an Extreme Style, Philadelphie, University of Pennsylvania Press, 2011 et Luis Alvarez, The Power of the Zoot. Youth Culture and Resistance during World War II, University of California Press, 2008. 
(...). Ils feraient mieux de se tenir tranquilles chez eux au lieu de se discréditer. Tu ne verras pas un Congolais tenir un balai ou juché sur une benne $e^{30}$." Djo Balard peut déclarer : "Le jean, c'est pour les mécaniciens, mon corps ne supporte pas le jean ${ }^{31}$." Si le discours est moins brutal, l'apparence du travailleur immigré vêtu de fripes reste un repoussoir. Quantité d'expressions doublent les vêtements. Outre le fait d'“aller à la chaîne", les sapeurs vont "à la mine" échanger des vêtements et quand ils "pointent", c'est qu'ils présentent leurs "gammes" en public. Et alors que se développe un discours contre les immigrés "assistés", quelques-uns osent en jouer, tel ce sapeur qui, dans dans une ultime subversion, s'est surnommé "Chômeur de luxe".

\section{Vestimentologie}

Apparaître comme "bien éduqué" est essentiel dans un contexte où l'immigré - comme le colonisé - est socialement déclassé. L'élégance est aussi de mots. Une des fiertés du Congo a été longtemps le fort taux d'alphabétisation; les sapeurs affichent leur maittrise de la langue française. Leurs noms revendiquent ce statut : Jocelyn Armel se fait appeler "le Bachelor" du fait de sa licence, Henri Blaise Nguebeyi, "Docteur", et tous se donnent volontiers du "professeur" ou du "maitre".

À observer leurs réunions, s'habiller c'est autant manipuler des mots que des tissus. "J'exaspère le français" dit l'un d'eux dans une formule radicale et ambiguë $\ddot{e n}^{32}$ : il s'agit bien de pousser la langue à bout pour en faire un instrument culturel et social. À la différence des subcultures et de leur argot (calo, verlan... ${ }^{33}$ ), les sapeurs, s'ils ont un argot fait de lari et de lingala, jouent la surintégration par une langue très soutenue qui, en se riant d'elle-même,

Le Bachelor en couturier, boutique Connivences, 2010. (c) Manuel Charpy, Kevin NGomsik et Pablo Grand-Mourcel

légifère et fixe une grammaire du vêtement ${ }^{34}$. Pour le sapologue, les "vers sont de lin, de soie, de tissus précieux et colorés ${ }^{35}$ ". En sémiologues du vêtement, les sapeurs ne cessent de dérouler un discours à la fois riche et creux, démonstration d'une maîtrise rhétorique et parodie de la langue savante. Ces dernières années, un débat a éclos entre "sapelogues" et "sapologues", querelle "scientifique" naturellement sans fin, qui place la sape dans le champ des sciences humaines. "Un sapelogue, écrit Mbouela, est (...) celui qui connaît la griffologie, la vestimentologie, l'harmonisation et la trilogie des couleurs achevées et inachevées ${ }^{36}$." Cette langue qui forge des néologismes comme la culture scientifique et la culture politico-religieuse congolaise (matswanisme, kimbanguiste, kakisme...) - la Sape jouant à se présen- 
ter comme une religion - sape par avance toutes les analyses surplombantes.

\section{"D'abord les habits, les courses après"}

La subversion des sapeurs repose aussi sur celle de l'argent. Depuis les années 1980, ils déclarent dépenser entre 50 et $75 \%$ de leurs revenus en sape et sont souvent endettés auprès des boutiques de luxe. "Moi, tout ce que je gagne va dans la sape, déclare l'un d'eux. Je gagne 1800 euros par mois, et j'en dépense 1 400. D'abord les habits, les courses après ${ }^{37}$ !" Certains sapeurs possèdent aujourd'hui plus de 200 "complets". Ces dépenses sont jugées anormales par la société française comme congolaise. On attend de l'immigré épargne et frugalité et des dépenses, en signe d'intégration, conformes à celles de la classe ouvrière française qui place le logement, la voiture, la nourriture et les loisirs avant les vêtements. En renversant les budgets par des dépenses somptuaires - près de 1000 euros dans une paire de chaussures -, les sapeurs vont à l'encontre des valeurs de tempérance inculquées à la classe ouvrière depuis le XIX siècle. Certains sapeurs, depuis les années 2000, non seulement affichent les marques de luxe mais laissent les prix visibles, à la manière des zooters avec leurs chaînes de portefeuille démesurées. Rien de surprenant à voir de jeunes sapeurs emprunter quelques signes au rap bling-bling.

Ces dépenses sont rendues possibles par une économie réduite. La Maison des étudiants du Congo, ironiquement appelée la MEC, a longtemps offert des loyers ridicules ${ }^{38}$. D'autres ont fait le choix de faire les "3-8" - roulement dans un lit - ou de s'entasser dans des studios et des chambres de bonne des beaux quartiers. Avec le temps, ils sont partis en banlieue. Quelques-uns pratiquent, dans les années 1980, le "Raymond Barre", technique de blocage des compteurs électriques en hommage ironique aux politiques d'austérité, le "boa", qui consiste à se faufiler dans les tourniquets du métro, la "dette coloniale", parfois le vol de vêtements pour les revendre...

La "vesture" des sapeurs les place du côté du tertiaire. Ils sont en effet nombreux à travailler dans de petits commerces - téléphonie, restauration... - et même ceux qui sont caristes ou ambulanciers font aussi l'import-export de voitures ou d'informatique d'occasion. D'autres encore sont vigiles, travail acceptable car de représentation. Les rares qui réussissent dans la mode comme Bachelor ou Djo Balard le font savoir. Ce dernier, associé à John Foster, fait chanter à son mariage: "John Foster, chaussures de luxe (...) / Djo Balard, le roi de la sape, invite hommes et La condamnation porte sur le gaspillage et renvoie les sapeurs à des origines bourgeoises largement imaginaires.

femmes chez John Foster / (...) vous trouverez le dernier parfum Djo Balard by John Foster et du bon champagne / (...) Le règlement intérieur exige d'être bien chaussé / et bien parfumé39." Si les vêtements et les chansons célèbrent l'entreprenariat, il ne s'agit pas pour autant de se fondre dans la masse des hommes d'affaires : cravate nouée en ceinture, kilt, ou deerstalker maintiennent l'excentricité.

Il ne s'agit pas ici d'analyser les "descentes" - retours - en "gammes complètes" au Congo, étape initiatique d'une vie d'aventurier ${ }^{40}$. Mais, de fait, une double assignation pèse sur les sapeurs, de la société dite "d'accueil" comme de départ. La double absence est une double contrainte ${ }^{41}$. Leurs dépenses somptuaires sont ainsi une provocation à légard à la fois de la société française et de la société congolaise, cette dernière attendant d'eux qu'ils souhaitent leurs compatriotes ${ }^{42}$. Si les retours des "Parisiens" sont orchestrés comme des consécrations, la violence des commentateurs congolais dit le caractère subversif de ces pratiques. Les 
condamnations portent sur le défaut - prétendu - de solidarité. La sapologie des "Parisiens", "cirque animé par une bande de clowns", serait un "fléau à combattre ${ }^{43}$ ". Des textes plus sophistiqués démontent la filiation avec Matsoua, considèrent avec condescendance la sape comme "normale" pour la jeunesse mais condamnent les "licenciés, maîtrisards, et parfois docteurs mais n'ayant aucun boulot" qui, entretenus par la famille, "brûlent l'argent de la popote pour s'acheter du Yoshi Yamamoto"4". La condamnation porte sur le gaspillage et renvoie les sapeurs à des origines bourgeoises largement imaginaires. Elle porte aussi sur lâge : la sape des vieux choque plus encore. Économistes et sociologues eux-mêmes peuvent être d'une grande violence, reconnaissant la dimension politique de la Sape mais en condamnant les "excès", soit son cœur même ${ }^{45}$.

\section{Allures et refus de l'invisibilité}

Dans le panthéon des sapeurs trônent aussi les musiciens, des groupes de rumba des années 1960 à Papa Wenba. Les sapeurs, dans leur goût des hétéronymes, se présentent eux-mêmes comme des "ambianceurs", des "saltimbanques", captant ainsi l'excentricité, le prestige et la publicité des musiciens et des couturiers. Car il s'agit bien de visibilité. À l'obligation de discrétion faite aux immigrés, les sapeurs opposent une visibilité maximum. Contrepartie de leur faible investissement dans l'immobilier, ils investissent l'espace public par le vêtement. Ils ont ainsi créé une géographie parisienne de micro-espaces de réunions, de monstrations ou d'exhibitions. Des années 1970 aux années 1990, c'est place de la République, à côté de la MEC et du Carreau du temple, puis, après 1977, la piazza de Beaubourg. Dès les années 1970, ils "démarchent" les Grands Boulevards, parfois jusqu’à la Chaussée d'Antin, s'emparant d'une scène de la mode inventée par les dandys du XIX ${ }^{\text {e }}$ siècle. Ils font ainsi des trottoirs des podiums. Un promoteur de la sape peut dire que le sapeur "est un acteur de grandes scènes, il est Molière, il est Sinatra ${ }^{46 "}$. Avec la fermeture de la MEC au milieu des années 1980, le centre de gravité des sapeurs se déplace vers les commerces - coiffure, téléphonie... - de Château-d'Eau et vers Château-Rouge où les sapeurs se retrouvent pour un "14 mètres" devant Connivences installé là depuis 2005. En parallèle, depuis les années 1980, ils se donnent à voir dans les espaces du luxe, autour de l'Opéra et sur les Champs-Élysées, dans des boutiques comme Weston. En naviguant entre ces espaces, ils démontrent que la Sape fonctionne aussi bien dans les territoires les plus huppés où ils choquent par leur élégance usurpée que dans les espaces populaires où ils contrastent. Et ils sont d'autant plus visibles dans la "ville-mode" qu'ils sont vêtus de couleurs dans un paysage vestimentaire, comme ils le notent eux-mêmes en baudelairiens, "funèbre". La "danse des griffes" dit le désir de visibilité : elle consiste pour partie à exhiber publiquement l'intérieur - hauts de chaussettes, boutonnières, doublures...

En outre, une série d'espaces sont investis de façon éphémère. Après les boîtes de nuit des années $1980^{47}$, églises et temples de la banlieue, pour les mariages et les funérailles et certains dimanches, sont devenus des lieux de réunions. Les captations vidéo rediffusées sur la Toile permettent de faire des rues vides de banlieue des boulevards et des podiums. Cette manière d'investir l'espace est en miroir - de ceux qu'on traverse -, les espaces de Paris et Brazzaville se répondant. Ainsi, alors que la brasserie Le Royal-Strasbourg était surnommé "Chez Lende", un des cafés de Bacongo était appelé "Le Royal-Strasbourg". Rien de surprenant au fait qu'un ticket du métro parisien serve de billet d'entrée dans les soirées de sapeurs brazzavillois.

Paris est ainsi à la fois le lieu de la parade, l'espace habité et la "ville-mode", lieu mythique. Les sapeurs, comme les zooters et les barjots installés entre les portes de Paris et le Drugstore des Champs-Élysées, inventent une manière de faire corps, d'appa- 
raître en public et de s'approprier une ville toujours plus hostile à la déambulation improductive.

Cet affichage dans l'espace public connaitt un redoublement médiatique. L'échange autour du vêtement - on "parle chiffons" des heures durant rue de Panama - est autant un ciment qu'une médiatisation. Les sapeurs eux-mêmes se transforment en marques, avec accroches publicitaires et parodiques : le Docteur Liman "soigne les apparences", le Bachelor "fait chanter les couleurs", Chômeur de luxe est une "beauté numérique"... Depuis les années 1980, de nombreuses cérémonies - des soirées d'élections aux funérailles en passant par les mariages - se font au son de la rumba congolaise. Si Papa Wenba avec ses fourrures tropicales est le plus exposé, de nombreux musiciens comme Stervos Niarcos et Rapha Bounzeki ${ }^{48}$ font l'hagiographie des sapeurs et sont émaillées de noms de marques de luxe et de boutiques dans lesquelles les sapeurs travaillent ${ }^{49}$.

Cette médiatisation connaît un second souffle sur Internet. Des chansons comme Danses des griffes sont captées par les sapeurs ou par des photographes et vidéastes payés pour l'occasion, et sont diffusées sur les blogs et les réseaux sociaux ${ }^{50}$. Tous sont de plus en plus maîtres de leur médiatisation, certains ayant toujours à portée de main leur revue de presse et relayant défilés, soirées, reportages, romans et expositions ${ }^{51}$. Les sapeurs tendent ainsi un miroir réfléchissant à la société française - les rares articles de sciences humaines n'échappent pas à ce libre recyclage ${ }^{52}$.

\section{Conclusion}

Les sapeurs ne se contentent pas de saper le statut d'immigré, ils sapent aussi les catégories, en leur échappant, de contre-culture ou de sous-culture. S'ils rejettent un travail physique, s'ils jouent avec les hiérarchies sociales, s'ils investissent des territoires publics, s'ils refusent enfin l'ordinaire des apparences - et une histoire globale de ces usages du vêtement en migration reste à écrire -, autant de caractéristiques d'une subculture ${ }^{53}$, ils ont en même temps toutes les caractéristiques d'une culture : racines revendiquées dans une histoire ancienne, discours sur ses pratiques, surinvestissement de la maîtrise de la langue, de lélégance et des bonnes manières... En ce sens, la Sape est une des nombreuses formes, à la fois populaire et savante, des braconnages culturels de pratiques comme de discours.

Détournements de la mode, la Sape emmène les marques et le luxe vers des périphéries géographiques, sociales et culturelles. C'est aussi qu'elle est indissociable d'une double situation de domination, celle des colonisés et celle des immigrés noirs en France. Elle n'est pas pour autant un simple masque blanc grimaçant. La polysémie du terme qui fait sa fortune - détruire les bases et s'habiller avec grande élégance - est une réponse complexe au poids de l'histoire, comme à celui qui pèse sur l'immigré et l'émigré. 